\title{
Tunable Stiffness And Damping Isolator Based On Modified Skyhook Control Method
}

\author{
Zheng Huiming ${ }^{1, a}$, Liang PeiQiong ${ }^{2, b}$ and Xiang $\mathrm{Yu}^{3, \mathrm{c}}$ \\ ${ }^{1}$ Department of Mechanics, Huazhong University of Science and Technology, Wuhan, Hubei \\ Province 430074, China \\ ${ }^{2}$ Guangxi Key Laboratory of Automobile Components and Vehicle Technology, Guangxi University \\ of Science and Technology, Liuzhou, Guangxi Province, 545006, China \\ ahuiming121212@hust.edu.cn; ${ }^{b}$ 2003010187@hust.edu.cn; '1037271105@qq.com
}

Keywords: modified skyhook method, stiffness and damping control , damping, vibration

\begin{abstract}
The original damping skyhook (SK) vibration control method needs to determine the absolute velocity of the sprung mass, however, the absolute velocity is impossible to measure for a running vehicle. The modified damping skyhook method (MSK) instead uses the derivation of acceleration of the sprung mass to indirectly determine the direction of the sprung mass absolute velocity. A one-degree-of-freedom quarter-car model with combined tunable stiffness and damping vibration isolator based on SK and MSK control method is developed.The obtained results show that ,for only damping control, the MSK and SK methods have a good vibration control efficacy only in acceleration control under enough high damping whereas for combined tunable damping and stiffness control, the MSK and SK methods have significant vibration control efficacy both in relative displacement and acceleration. Moreover, the MSK method performs better control effect than SK under sinusoid and random excitations. Therefore MSK method is feasible in vehicle suspension system design because of its simplicity and good vibration control efficacy in both relative displacement and acceleration.
\end{abstract}

\section{Introduction}

For vehicle suspension system design, it is always challenging to maintain simultaneously a high standard of ride, handling, and body attitude control under all driving conditions. The problems stem from the wide range of operating conditions created by varying road conditions, vehicle speed, and load. A good suspension system should provide good vibration isolation, i.e. small acceleration of the body mass, and a small "rattle space", which is the maximal allowable relative displacement between the vehicle body and various suspension components[1].The ideal goal of an optimal suspension is to minimize the sprung mass relative displacement and acceleration. However, these two criteria are in conflict. In general, a suspension system with a small relative displacement corresponds to a high sprung mass acceleration, and a large relative displacement corresponds to a low sprung mass acceleration.

Semiactive (SA)vibration isolation systems have been intensively studied and widely used in reducing the vibration of vehicle suspension system. One common SA vibration isolation system is the tunable damping system, which has been proven to be an effective vibration control technique[2] . Besides, tuning the stiffness of the vibration isolation system is another method in a SA system. A few researchers have proposed some vibration isolation systems with tunable stiffness to prove their effectiveness[3 ].Several semi-active control methods have been proposed. Shen Y. investigated the modified skyhook control (MSK)[4].The original skyhook(SK) method is applied to set the vibration control strategy in a way that the damper is switched to a high damping ratio when the direction of absolute velocity and the relative velocity are same, and a low damping value otherwise[5]. The SK method needs to determine the absolute velocity of the sprung mass, however, the absolute velocity $\dot{x}_{s}$ is impossible to measure for a running vehicle. In general, we can only measure the acceleration of the sprung mass. Hedrick et al. suggested that the absolute 
sprung mass velocity $\dot{x}_{s}$ cannot be estimated in an exponentially stable manner[6]. It could theoretically be obtained by integrating the acceleration of the sprung mass and passing the result through a high-pass filter to re move the direct current offset. In practice, this is difficult to do because the acceleration offset is not constant and the initial condition of the integral is hard to be determined. For these reasons, Shen Y. developed the modified skyhook (MSK) control method based on the case that for a linear vibration system with a sinusoidal input, the phase difference between the absolute velocity and derivation of acceleration is $\pi$ [4]. The MSK method does not require the absolute velocity of the sprung mass, but instead uses the derivation of acceleration of the sprung mass to indirectly determine the direction of the sprung mass absolute velocity.

The MSK method is applied to set the vibration control strategy in a way to only switch damping. In this paper, a combined tunable stiffness and damping on-off control based on damping SK and MSK control method is present.For a variable stiffness or high damping on-off vibration, the dynamical response may be nonlinear. A one-degree-of-freedom (1DOF) quarter-car model with vibration isolator under different ground base payload has been adopted to study the efficacy of the SK and MSK control methods .

\section{Control Strategies}

In the simplest approach to evaluate the vibration control efficiency of different control strategies, a one-degree-of-freedom (1DOF) quarter-car model, as shown in Figure 1,has been adopted. Magnetorheological elastomer (MRE) acts as semi-active system actuators for its rapid response to the applied magnetic field and their compact size. $m$ is the mass of the payload; $c$ and $k$ are the damping coefficient and the stiffness of the isolator, respectively; $x_{\mathrm{S}}$ and $y$ are the displacements of the payload and the ground base, respectively. The equation of motion for the system is as follows:

$$
m \ddot{x}_{s}+F_{d}+F_{k}=0
$$

where (..) and (.) mean $\mathrm{d}^{2} / \mathrm{d} t^{2}$ and $\mathrm{d} / \mathrm{d} t$, damping force $F_{d}=c\left(\dot{x}_{s}-\dot{y}\right)$ and spring force $F_{k}=k\left(x_{s}-y\right) \cdot k$ is the elastic coefficient , $c=2 \zeta \sqrt{\mathrm{km}}, \zeta$ is viscous damping ratio.

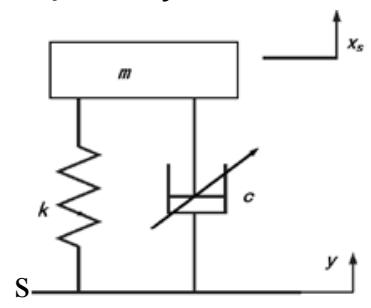

Fig.1. 1DOF suspension system

Without control, $k=k_{n}, k_{n}=\left(2 \pi f_{n}\right)^{2} m, f_{\mathrm{n}}$ is natural frequency $\zeta=\zeta_{0}$. According to the original skyhook working principle, the semi-active on-off damping control law is[4]

$$
\varsigma=\left\{\begin{array}{l}
\varsigma_{\max } \text {;if } \dot{x}_{s} \dot{x}_{r} \geq 0 \\
\text { else } \\
0
\end{array}\right.
$$

Modified skyhook method(MSK) [4]

$$
\varsigma=\left\{\begin{array}{l}
\varsigma_{\max } ; \text { if } \dddot{x}_{s} \dot{x}_{r} \leq 0 \\
\text { else } \\
0
\end{array}\right.
$$

In this paper, we propose combining stiffness control and damping control laws for MRE-based vibration isolator as follows.

Skyhook method(SK) 


$$
\left\{\begin{array}{l}
k=k_{\max }=(1-s) k_{n} \text { and } \zeta=\zeta_{\max }=\zeta_{0} ; \text { if } \dot{x}_{s} \dot{x}_{r}>0 \\
\text { else } \\
k=k_{\min }=(1+s) k_{\min } \quad \text { and } \zeta=\zeta_{\min }
\end{array}\right.
$$

Modified skyhook method(MSK)

$$
\left\{\begin{array}{l}
k=k_{\max }=(1-s) k_{n} \text { and } \zeta=\zeta_{\max }=\zeta_{0} ; \text { if } \dddot{x}_{s} \dot{x}_{r}<0 \\
\text { else } \\
k=k_{\min }=(1+s) k_{\min } \text { and } \zeta=0
\end{array}\right.
$$

where $s$ is tunable stiffness ratio,the jerk $\dddot{x}_{s}$ of the sprung mass can be obtained by differentiating the filtered acceleration of the sprung mass. Obviously for a linear vibration system with a sinusoidal input, the phase difference between the jerk $\dddot{x}_{s}$ and $\dot{x}_{s}$ is $\pi$ in this case as discussed in [4]. This control algorithm makes the MSK method easier to implement in a practical system without using a complex observer. Note that the phase difference is not always $\pi$ in other cases.

Variable Damping Control. Since the nature frequency of the vibration isolation system is $5 \mathrm{~Hz}$, the sinusoid excitation frequency in the investigation was chosen 5Hz. Figs.2(a,b) give the steady-state response to a sinusoid base excitation of a suspension system with $\zeta=0.6$. The relative displacement amplitudes $\mathrm{Xr}$ and acceleration RMS values of the response of the payload with $\zeta=0.3,0.4,0.6$ are tabulated in Table 1 .

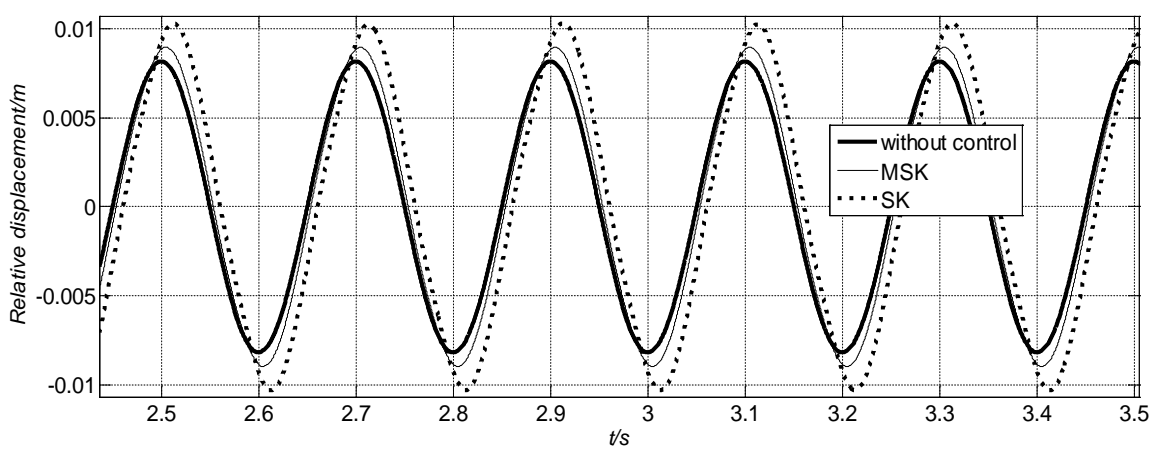

(a) Relative displacement

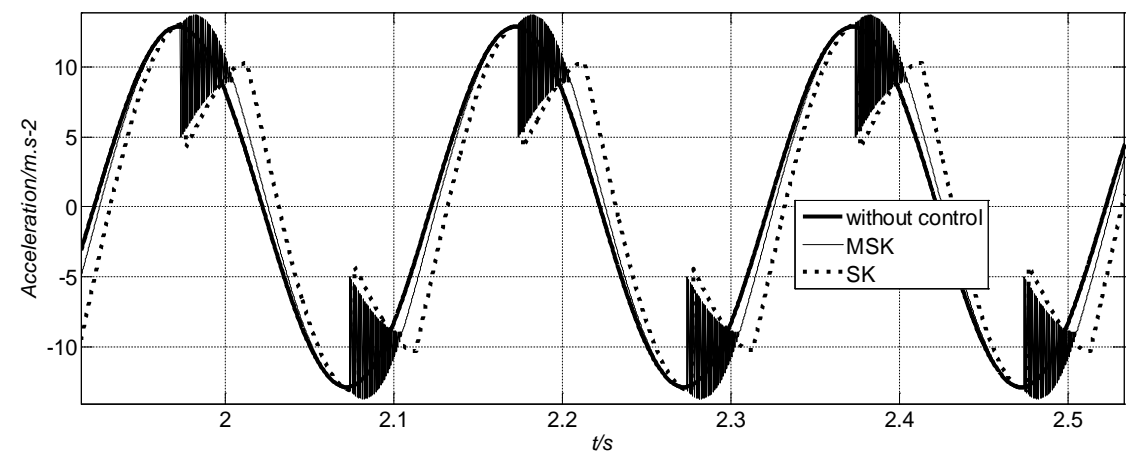

(b) Acceleration. $\zeta=0.6$

Fig.2 Response to a sinusoid base excitation at $f=5 \mathrm{~Hz}$ 
Table 1 Relative displacement and acceleration RMS values at frequency $\mathrm{fn}=5 \mathrm{~Hz}$

\begin{tabular}{c|c|c|c}
\hline$\zeta$ & 0.3 & 0.4 & 0.6 \\
\hline \multicolumn{2}{|c|}{$X \mathrm{r}(\mathrm{m})$} & 0.0082 \\
\hline Without control & 0.0165 & 0.0123 & 0.0090 \\
\hline MSK & 0.0168 & 0.0128 & 0.0103 \\
\hline SK & 0.0171 & 0.0134 & 9.0669 \\
\hline \multicolumn{2}{|c|}{ Acceleration $\left(\mathrm{ms}^{-2}\right)$} \\
\hline Without control & 13.5386 & 11.1500 & 8.9729 \\
\hline MSK & 13.4504 & 11.0389 & 8.4348 \\
\hline SK & 13.2995 & 10.7737 & .
\end{tabular}

From Figs.2(a,b) and Table 1,it can be seen that relative displacement response is approximate sinusoid wave but acceleration response is not approximate sinusoid wave especially in the vicinity of peak range. For damping ratio $\zeta$ is smaller than 0.3, MSK and SK control effects have very little difference and are very close to the case of without control. For enough high damping ratio, such as $\zeta=0.6$.In all cases, the SK control method has the lowest RMS acceleration when compared with the other control methods while the case of without control has the lowest relative displacement Xr. MSK and SK perform better control in acceleration but increase the relative displacement. As damping is enough high,MSK makes an obvious difference from SK control. However, in general, loss factor of MRE material is not enough high up to 0.6, MSK and SK with only damping on-off control perform very close control effect. Therefore, using jerk feedback control instead of velocity is feasible as described in the paper[4].

Combined Tunable Damping and Stiffness Control. In general, for MER material, the damping ratio is below 0.05[7], therefore, the tunable stiffness SK and MSK control methods with small damping are investigated in the following.

Figs.3(a,b) give the steady-state response to a sinusoid base excitation of frequency $5 \mathrm{~Hz}$ when combined tunable damping and stiffness MSK and SK control methods are applied. In this case ,stiffness ratio $s$ is chosen as 0.3 with small constant damping ratio $\zeta=0.05$. The relative displacement amplitudes and acceleration RMS values of the response of the payload with $s=0.1,0.2,0.3$ and $\zeta=0.05$ are tabulated in Table 2 .

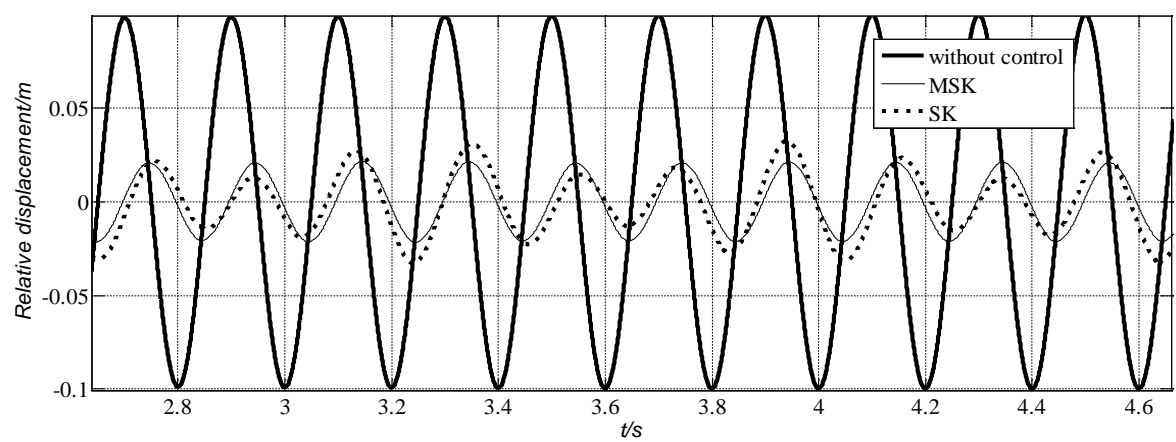

(a)Relative displacement

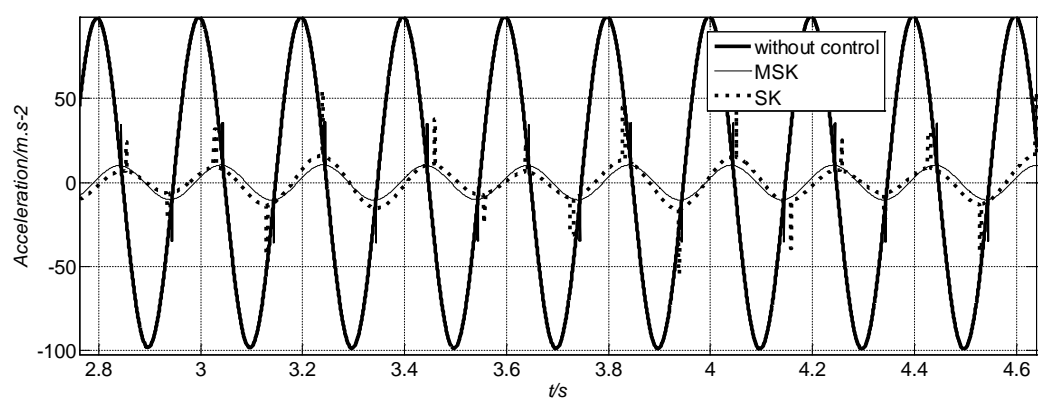

(b) Acceleration ( $s=0.3, \zeta=0.05)$

Fig.3 Response to a sinusoid base excitation at $f=5 \mathrm{~Hz}$ 
From Figs.3 and Table 2, it can be seen that combined tunable stiffness and damping SK and MSK control methods obtain significant reduction in both relative displacement and acceleration RSM values. As stiffness tunable ratio $s$ increases, the vibration reduction increases. From the results obtained from above investigation, small damping on-off control has little effect on vibration, this implies that on-off stiffness shift plays a dominant role over damping on-off shift. For variable stiffness system, the vibration responses are nonlinear, the response of relative displacement and acceleration is not accurate sinusoid wave, especially for acceleration responses and relative displacement of SK method as shown in Figs.3, the phase difference between the jerk $\dddot{x}_{s}$ and $\dot{x}_{s}$ is not always $\pi$. MSK and SK control effects are different.MSK method performs better control than SK method in both relative displacement and acceleration.

Table 2 Relative displacement and acceleration RMS values at frequency $\mathrm{fn}=5 \mathrm{~Hz}, \zeta=0.05$

\begin{tabular}{c|c|c|c}
\hline$s$ & 0.1 & 0.2 & 0.3 \\
\hline \multicolumn{2}{|c|}{$X \mathrm{r}(\mathrm{m})$} & 0.0995 & 0.0995 \\
\hline Without control & 0.0995 & 0.0323 & 0.0234 \\
\hline MSK & 0.0515 & 0.0398 & 0.0336 \\
\hline SK & 0.0551 & Acceleration $\left(\mathrm{ms}^{-2}\right)$ \\
\hline \multicolumn{5}{c|}{} & 65.4936 & 65.4936 \\
\hline Without control & 65.4936 & 14.2557 & 8.4291 \\
\hline MSK & 30.0941 & 15.4046 & 10.3494 \\
\hline SK & 32.1140 &
\end{tabular}

Response to Random Base Excitations. In general, a suspension system with one control law will be subjected to various kinds of base excitation loads. It is desirable for one kind control law can perform better control under several typical base excitations. To further evaluate control effect of the combined tunable damping and stiffness SK and MSK methods,the responses of the payload to random base excitations were simulated with the same parameters used in the previous discussion.

Fig. 4 shows the time history of a random base excitation.The random signals are the uniform distribution signals filtered by a band-pass filter. Since the vibration of the payload around nature frequency is the severest and the isolation efficiency increases with increasing of the frequency, the frequency range of the random excitation was chosen around the resonant frequency ranging from 0 to $10 \mathrm{~Hz}$ and the RMS value of excitation is $7.24 \mathrm{e}-005 \mathrm{~m}$.

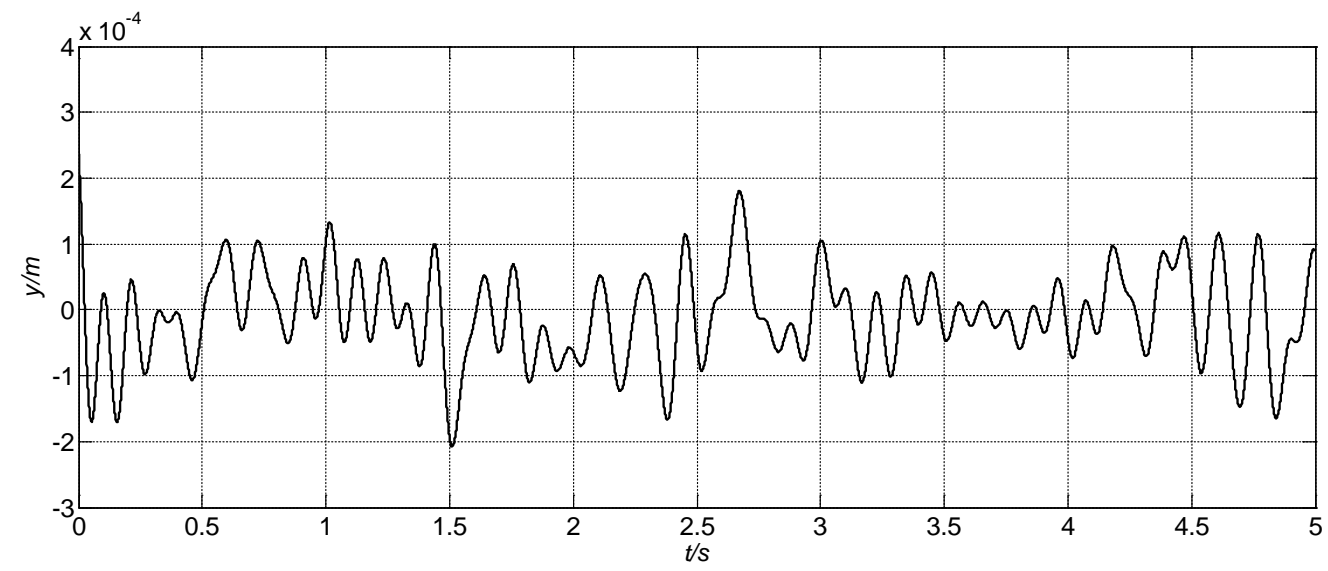

Fig.4 Time history of random excitation 
Table 3 RMS values of relative displacement and acceleration to random excitation, $\zeta=0.05$

\begin{tabular}{|c|c|c|c|}
\hline$S$ & 0.1 & 0.2 & 0.3 \\
\hline \multicolumn{4}{|c|}{$X \mathrm{r}\left(10^{-3} \mathrm{~m}\right)$} \\
\hline Without control & 0.2074 & 0.2074 & 0.2074 \\
\hline MSK & 0.1945 & 0.1728 & 0.1351 \\
\hline SK & 0.1960 & 0.1883 & 0.1558 \\
\hline \multicolumn{4}{|c|}{ Acceleration $\left(\mathrm{ms}^{-2}\right)$} \\
\hline Without control & 0.2109 & 0.2109 & 0.2109 \\
\hline MSK & 0.1572 & 0.1184 & 0.0797 \\
\hline SK & 0.1655 & 0.1423 & 0.1024 \\
\hline
\end{tabular}

Figs.5 give the response to a random base excitation when combined tunable damping and stiffness SK and MSK control methods are applied with $\mathrm{s}=0.3$ and $\zeta=0.05$.The relative displacement and acceleration RMS values of the response with $s=0.1,0.2,0.3$ with $\zeta=0.05$ are also tabulated in Table 3. The obtained results from Fig.5 and Table 3, for s=0.1,0.2 and 0.3, MSK and SK methods can reduce simultaneously both relative displacement and acceleration. As $s$ increases, the vibration reduction becomes significant. Similar to sinusoid excitation, MSK performs better control than SK method.

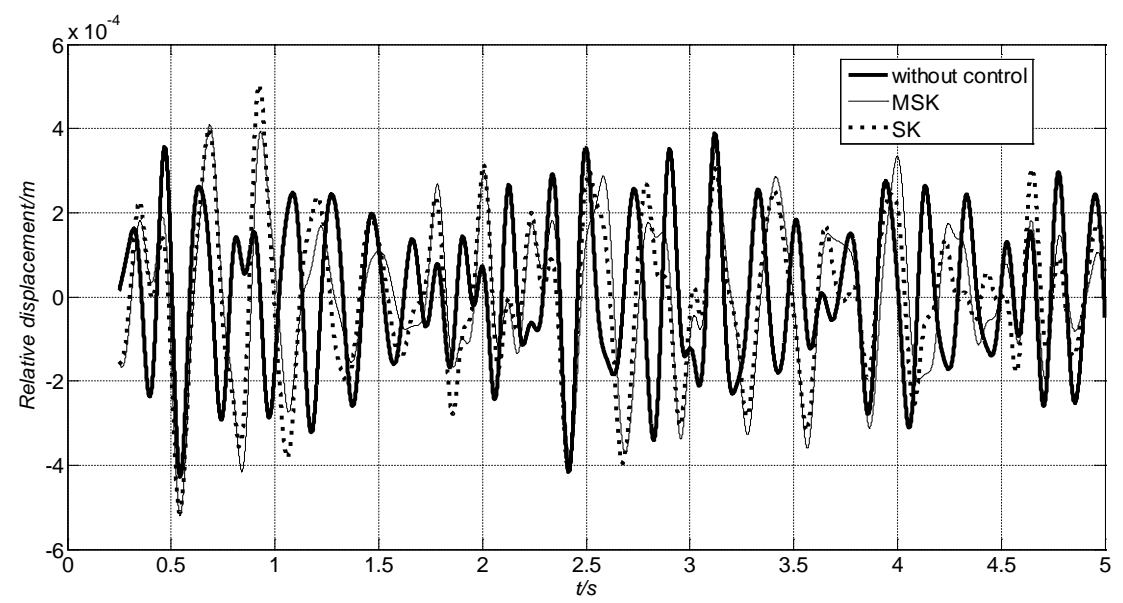

(a) Relative displacement

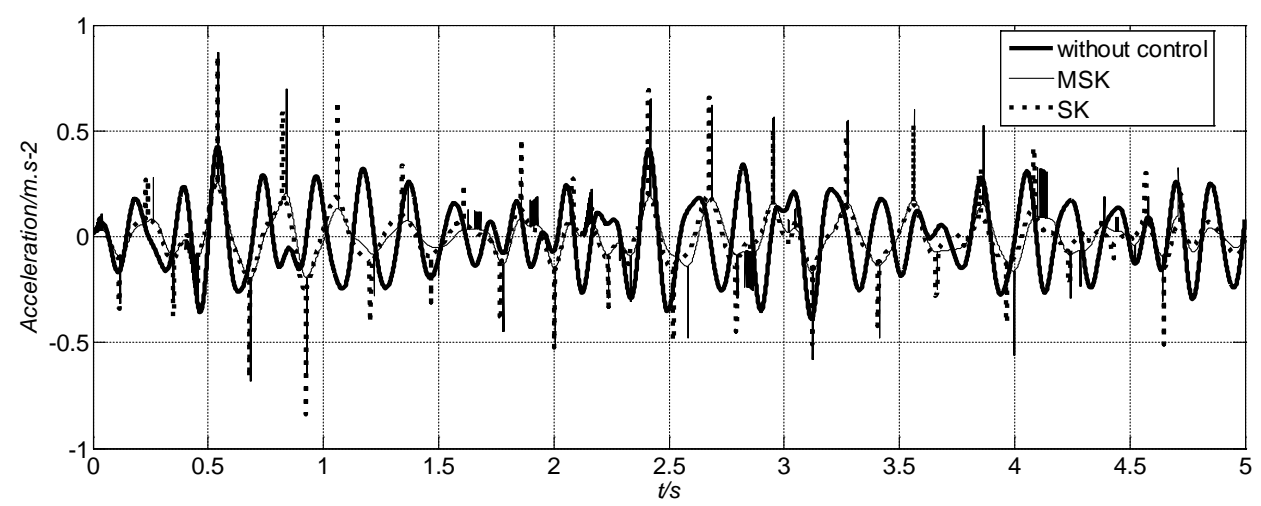

(b) Acceleration $(\mathrm{s}=0.3, \zeta=0.05)$

Fig. 5 Response to a sinusoid base excitation at $f=5 \mathrm{~Hz}$

\section{Conclusions}

The vibration control efficacy of the tunable stiffness and damping isolator based on modified skyhook control method is investigated. The SK method needs to determine the absolute velocity of the sprung mass but MSK method instead uses the derivation of acceleration of the sprung mass to indirectly determine the direction of the sprung mass absolute velocity. For only damping on-off 
control, the MSK and SK methods has a good vibration control efficacy only in acceleration control under enough high damping. For combined tunable damping and stiffness control, the MSK and SK methods have significant vibration control efficacy both in relative displacement and acceleration. Moreover, the MSK performs better control effect than SK under sinusoid and random excitations. Therefore, MSK method is feasible in vehicle suspension system design because of its simplicity and good vibration control efficacy to simultaneously minimize the sprung mass relative displacement and acceleration.

\section{Acknowledgment}

This work is supported by Open fund project (No.2012KFZD01) of Guangxi Key Laboratory of Automobile Components and Vehicle Technology,Guangxi University of Science and Technology , Guangxi province,China.

\section{References}

[1] Hrovat D., Margolis D. L. and Hubbard, M(1988)An approach toward the optimal semi-active suspension.Journal of Dynamic Systems, Measurement, and Control 110, 288-296.

[2] Yu M, Dong, XM, Choi and Liao CR (2009) Human simulated intelligent control of vehicle suspension system with MR dampers. Journal of Sound and Vibration 319(3-5):753-767.

[3] Liu YQ, Matsuhisa H and Utsuno H (2008) Semi-active vibration isolation system with variable stiffness and damping control. Journal of Sound and Vibration 313(1-2):16-28.

[4] Shen Y, Golnaraghi M. F. and Heppler G. R(2006)Semi-active Vibration Control Schemes for Suspension Systems Using Magnetorheological Dampers. Journal of Vibration and Control 12: 3-24.

[5] Alanoly J. and Sankar, S.(1987)A new concept in semi-active vibration isolation. Journal of Mechanisms, Transition and Automation in Design 109(2), 242-247.

[6] Hedrick J. K., Rajamani, R. and Yi, K.(1994)Observer design for electronic suspension applications. Vehicle System Dynamics 23, 413-440.

[7] R. Li and L. Z. Sun (2011)Dynamic mechanical behavior of magnetorheological nanocomposites filled with carbon nanotubes (CNMRE) Applied Physics Letters 99:131912 